https://doi.org/10.7559/gestaoedesenvolvimento.2019.376

Data de receção: 11/04/2019 Data de aceitação: 12/07/2019

\title{
MOTIVAÇÕES PARA ADOÇÃO DE BUSINESS ANALYTICS
}

\section{MOTIVATIONS FOR ADOPTION OF BUSINESS ANALYTICS}

\author{
Joana Fernandes ${ }^{1}$ orcid.org/0000-0003-4227-0423 \\ António Andrade ${ }^{2}$ orcid.org/0000-0001-8096-4720
}

\begin{abstract}
Resumo: Ainda que as novas formas de gestão já valorizem a adoção de ferramentas e algoritmos de análise de base de dados, estando, por sua vez, cada vez mais predispostas a utilizar análises em tempo real e preditivas, existe ainda alguma relutância por parte das gerações mais conservadoras para a sua adoção (Arasteh, Aliahmadi, Mahmoodi, \& Mohammadpour, 2011; Chen, Chiang, \& Storey, 2012).

Por conseguinte, o propósito da investigação será identificar as motivações para a adoção do Business Analytics em Portugal. Para este efeito foi realizado um estudo exploratório tendo como base o modelo desenvolvido por Venkatesh, Thong, \& Xu, (2012) a: Unified Theory of Acceptance and Use of Technology (UTAUT).

$O$ processo de recolha de dados foi realizado através de um inquérito por questionário online, cujo público alvo foi a população portuguesa. O total de respostas obtido foi de 102, sendo que sendo que $47,8 \%$ dos inquiridos se identificam como indivíduos do sexo Masculino e 52,2\% como sendo do sexo Feminino.

A análise de dados foi dividida em duas partes, numa primeira fase procurou-se identificar os determinantes de aceitação já pré identificados pela UTAUT, através de uma análise por componentes principais. Neste sentido, foi possível identificar quatro determinantes de aceitação: a Expectativa de Desempenho, a Expectativa de Esforço, as Condições Facilitadoras e a Intenção Comportamental.
\end{abstract}

\footnotetext{
${ }^{1}$ Católica Porto Business School

${ }^{2}$ CEGE, Católica Porto Business School. Email: aandrade@ porto.ucp.pt
} 
Na segunda parte procurou-se, através da utilização de modelo de regressão clássica, analisar as motivações na base da adoção do Business Analytics para cada um dos determinantes.

A análise permitiu ainda concluir, que a adoção é fortemente influenciada pela posição hierárquica. Ademais, concluiu-se, ainda, que o preço e a expectativa do retorno do investimento não foram considerados os maiores impeditivos na adoção do Business Analytics. No entanto, verificou-se que o não conhecimento do conceito de inteligência artificial é um dos principais condicionantes à adoção.

Palavras-chave: Business Analytics, Business Intelligence, UTAUT

Abstract: Business Analytics and Business Intelligence are an emerging trend in Management nowadays. However, despite the advantages perceived by some managers in the utilization of more predictive algorithms and tools, still exists some others that are more conservative about it.

Moreover, the literature identifies some advantages and limitations regarding the adoption of Business Analytics, nonetheless does not exists information respecting the adoption in the Portuguese Market (Arasteh et al., 2011; Chen et al., 2012).

Hence, the main goal of the study was to identify the reasons and motivations for the adoption of the Business Analytics in the Portuguese population. Thereby, in order to identify the acceptation determinants an exploratory study was carried based on a model suggested by Venkatesh et al., (2012), the Unified Theory of Acceptance and use of Technology (UTAUT).

The database information was obtained through an online survey questionnaire, and the universe of the research was 102 answers, with $47,8 \%$ had been of masculine sex and 52,2\% of the feminine one.

Additionally, through a principal components analysis we could identify four acceptation determinants; the Performance Expectancy, the Effort Expectancy, the Facilitating Conditions and the Behavioral Condition

Furthermore, using a classic regression analysis we were able to conclude that the motivations are depend on the hierarchical position 
of the individual and that the price does not influence the adoption as expected. However, the main conclusion was the identification of the lack of information of artificial intelligence as the main constraints.

Keywords: Business Analytics, Business Intelligence, UTAUT

\section{BUSINESS ANALYTICS E BUSINESS INTELLIGENCE}

\subsection{Morfologia dos Elementos}

A quarta revolução industrial abriu espaço para o aprofundamento da digitalização dos processos de gestão. A possibilidade de maximizar a eficiência e a competitividade através de uma definição de estratégias mais alinhadas com as necessidades intensificou o aumento do interesse por parte das empresas na exploração inteligente de dados (Cooper, 2012).

Todavia, antes de procurar as motivações para a adoção destas novas ferramentas é importante definir o Business Analytics e o Business Intelligence. Cooper, (2012) e Delen, (2014) caracterizaram o Business Analytics como a aplicação de técnicas e de modelos estatísticos na resolução de problemas complexos nas organizações/empresas, procurando fortificar as relações entre os intervenientes.

Por sua vez, o Business Intelligence, têm um foco mais imediato nas operações, visto que, para além da recolha e análise de dados, engloba ainda a criação de relatórios e a visualização dos dados, convocando em si conhecimentos de Querying, Data Mining e Online Analytics Processing (OLAP) (Santos \& Ramos, 2006).

\subsection{Conceitos e Propósitos}

Ainda que, as razões pelas quais todos os tipos de organizações investem em ferramentas de Business Analytics e Business Intelligence possam ser aceleradas pelo tipo de mercado ou a filosofia de gestão, existem, no entanto, fatores comuns entre elas (Acito \& Khatri, 2014; Ferreira \& Andrade, 2013).

Para as empresas, especificamente, os sistemas de gestão de base tecnológica que incorporem a vertente analytics são particularmente úteis. Desde a facilitação da gestão das relações com os clientes, trabalhadores e investidores; à identificação de transações fraudulentas e 
comportamentos inabituais; à melhoria do produto ou serviço; otimização das estratégias de marketing e campanhas publicitárias; minimização dos custos operacionais, de inventário e de alocação e, por último, a ajuda numa tomada de decisão mais célere, se necessário, mas sempre mais informada (Delen, 2014).

Todavia, um dos maiores impasses para a sua adoção é a inexperiência ou a visão limitada do conceito por parte dos gestores, pois é importante que estes não considerem as ferramentas apenas como uma calculadora ou como um depósito de informação que permite relatórios convencionais embora, talvez, mais próximos do tempo real (Appelbaum, Kogan, Vasarhelyi, \& Yan, 2017).

Evidentemente que a análise profunda de dados, estruturados ou não, cruzados a partir de múltiplas fontes, que é feita através de algoritmos muito sofisticados é um conceito relativamente recente, existindo, portanto, poucas pessoas no mercado capacitadas para tal (Delen, 2014).

McAfee, Brynjolfsson, Davenport, Patil, \& Barton (2012), identificaram a liderança, o talento do gestor, a tecnologia, a cultura da organização e a necessidade de suporte à tomada de decisão como os principais desafios para a implementação sem esquecer os determinantes éticos (Passos, 2017).

Delen (2014) identificou ainda mais cinco motivos, a par com o talento analítico do gestor, está ainda a cultura do mesmo, ou seja, os hábitos que foi criando ao longo do seu percurso profissional, e a impossibilidade de prever o retorno do investimento. Embora se saiba que na maioria dos casos a adoção das ferramentas gere um retorno positivo, pode acontecer que, por má implementação, ou fraco empenhamento das chefias, aconteça o contrário gerando perdas significativas para a empresa e, por conseguinte, um abandono destas ferramentas.

O preço das ferramentas necessárias para a implementação dos sistemas foi também considerado um outro entrave (Delen, 2014), muitas empresas preferem adotar os sistemas tradicionais de gestão a inovar dado o risco de não dominarem o seu potencial e identificarem eventuais custos escondidos. Por último, identificou ainda a segurança e a privacidade como fatores importantes e impeditivos na adoção do Business Analytics e do Business Intelligence. 


\subsection{A Tecnologia e a Gestão Organizacional}

As tecnologias de informação são importantes nas diferentes unidades económicas, todavia, é nos ecossistemas das empresas que nos apercebemos da melhor interação entre os equipamentos da organização e as pessoas, aumentando deste modo a sua complexidade (Ghosh, Gajar, \& Rai, 2013).

No momento atual, a grande maioria dos gestores das empresas está exposto a excessivas quantidades de informação, e têm acesso a múltiplas fontes de bases de dados internas e externas, que podem ou não conter dados estruturados (Kaye, 1995).

Deste modo, é importante que consiga não só gerir a parte operacional, isto é, os dados armazenados em estruturas devidamente definidas, como ainda os dados não estruturados provenientes, por exemplo, de plataformas sociais, ou do correio eletrónico (Amaral, 1994).

Além do mais, atualmente as empresas trabalham em mercados extremamente complexos e competitivos obrigando a que exista um elevado grau de precisão na tomada de decisão (Cordeiro \& Gouveia, 2018; Shuai, Yi- Fen Sue, \& Chyan Yang, 2007).

Um sistema de informação deve, deste modo, estar preparado para suportar todos os níveis de gestão e as diferentes especificidades de cada um, por isso é, normalmente, constituído por diversos subsistemas com características especificas para a sua finalidade (Mesquita, Faria, Gonçalves, \& Varajão, 2013).

Atualmente podemos considerar a informação como um dos ativos mais importantes para uma organização. Sendo utilizada por algumas organizações como uma forte aliada estratégica, indispensável na obtenção de vantagem competitiva (Amaral, 1994).

Porém, sabe-se que um dos principais problemas do mundo empresarial é a falha no entendimento entre aqueles que gerem e os que analisam. Com efeito, a informação deve ser a mais adequada à situação e às particularidades do utilizador, contudo, não existe nada que garanta que irá ser interpretada corretamente e usada de forma eficaz (Mesquita et al., 2013).

De acordo com Ransbotham \& Kiron (2017), existe uma capacidade de inovar maior quando as empresas implementam o Analytics, pois de 
facto ele identificou ainda quatro características facilitadoras desta inovação, apresentadas no quadro abaixo.

\section{Quadro I}

\section{Características Facilitadoras da Inovação}

Vantagem Comparativa: Verificou-se um aumento da vantagem comparativa devido a vários fatores, incluindo uma maior dispersão de análises dentro das empresas, em conjunto com uma melhor perceção das vantagens da mesma e ainda um foco mais forte em serviços especializados

Capacidade de Inovação: Verificou-se que organizações com forte capacidade analítica tem vantagem em inovar, desde criação de produtos/serviços novos a melhoria dos antigos;

Partilha de dados: Empresas que partilham dados internamente desenvolvem melhores análises que posteriormente ajuda na melhoria dos processos;

Smart Machines: As empresas analiticamente mais maduras usam inteligência artificial para assumir tarefas demoradas e standard libertando os gestores para questões estratégicas.

\section{METODOLOGIA DE INVESTIGAÇÃO}

\subsection{Contextualização}

A literatura evidencia um crescente aumento da procura por soluções de Business Analytics, no entanto, embora muitas empresas tenham aderido a estas novas ferramentas de gestão e análise de dados existe ainda alguma resistência à sua implementação (Arasteh et al., 2011).

Por conseguinte, o presente estudo irá procurar encontrar os fatores influenciadores da aceitação e do uso do analytics e da inteligência artificial, através da realização de estudo exploratório com base no modelo desenvolvido por Venkatesh, Thong, \& Xu, (2012) a: Unified Theory of Acceptance and Use of Technology (UTAUT).

\subsection{Objetivo da Investigação}

Como supramencionado o objetivo da investigação é a realização de um estudo exploratório através da análise das respostas dadas num inquérito por questionário.

$\mathrm{O}$ inquérito foi construído tendo como base as determinantes identificadas na UTAUT. Neste sentido, a validação da consistência 
interna foi realizada, apenas, através de uma análise semântica e por uma análise por painel de peritos.

O modelo irá utilizar, ainda, a idade, o sexo, a escolaridade, a experiência profissional (departamento e setor de atividade) como variáveis moderadoras.

Em síntese, o estudo procurará identificar os determinantes mais importantes na implementação do Business Analytics pelos indivíduos.

\subsection{Síntese da Fundamentação}

O Quadro II apresenta em síntese a fundamentação desenhada para a conceção do instrumento de recolha de dados.

\section{Quadro II}

\section{Revisão Esquemática}

\begin{tabular}{|c|c|c|}
\hline Determinantes & Resultados da Literatura & Autor(es) \\
\hline \multirow{10}{*}{$\begin{array}{l}\text { Expectativa de } \\
\text { Desempenho }\end{array}$} & $\begin{array}{l}\text { Que vantagens considera importantes aquando } \\
\text { da implementação do Business Analytics? }\end{array}$ & \multirow{10}{*}{$\begin{array}{l}\text { (Acito \& Khatri, } \\
\text { 2014); } \\
\text { (Arasteh et al., } \\
\text { 2011); } \\
\text { (Chen et al., 2012); } \\
\text { ("Coming of Age } \\
\text { Digitally: } \\
\text { Learning, } \\
\text { Leadership and } \\
\text { Legacy | Deloitte } \\
\text { Insights," 2018); } \\
\text { (Cooper, 2012); } \\
\text { (Delen, 2014); } \\
\text { (Lindgreen et al., } \\
\text { 2012) }\end{array}$} \\
\hline & $\begin{array}{l}\text { Que benefícios espera obter da introdução de } \\
\text { Business Analytics? }\end{array}$ & \\
\hline & $\begin{array}{l}\text { O BA disponibiliza informação que potencia } \\
\text { envolver mais o cliente. }\end{array}$ & \\
\hline & $\begin{array}{l}\text { O BA dá à empresa maior capacidade analítica, } \\
\text { o que implica adquirir maior competitividade. }\end{array}$ & \\
\hline & $\begin{array}{l}\text { O BA pode contribuir para a evolução da cultura } \\
\text { da empresa. }\end{array}$ & \\
\hline & $\begin{array}{l}\text { O BA ao reunir informação de uma diversidade } \\
\text { de fontes de dados, contribui para elevar o valor } \\
\text { para a empresa. }\end{array}$ & \\
\hline & 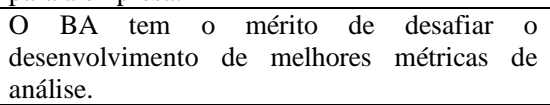 & \\
\hline & $\begin{array}{l}\text { Implementar o BA perspetiva à empresa ter } \\
\text { dados para melhorar o seu desempenho. }\end{array}$ & \\
\hline & $\begin{array}{l}\text { A introdução do BA é importante na captação e } \\
\text { manutenção de clientes. }\end{array}$ & \\
\hline & $\begin{array}{l}\text { A introdução do BA é importante na melhoria } \\
\text { das parcerias e comunicações com outras } \\
\text { empresas. }\end{array}$ & \\
\hline \multirow{2}{*}{$\begin{array}{l}\text { Cultura } \\
\text { Organizacional/ } \\
\text { Hábito }\end{array}$} & $\begin{array}{l}\text { Quão competente é a sua empresa na adoção } \\
\text { de tecnologias emergentes. }\end{array}$ & \multirow{2}{*}{ (Jarrahi, 2018) } \\
\hline & $\begin{array}{l}\text { Quão competente é a sua empresa em termos de } \\
\text { análise e gestão de dados. }\end{array}$ & \\
\hline
\end{tabular}




\begin{tabular}{|c|c|c|}
\hline & $\begin{array}{l}\text { Quão competente é a sua empresa em termos de } \\
\text { análise de dados avançadas. }\end{array}$ & \\
\hline & $\begin{array}{l}\text { Quão competente é a sua empresa na } \\
\text { valorização de uma cultura organizacional } \\
\text { aberta? }\end{array}$ & \\
\hline \multirow{4}{*}{$\begin{array}{l}\text { Condições } \\
\text { Facilitadoras/ } \\
\text { Intenção } \\
\text { Comportamental }\end{array}$} & $\begin{array}{l}\text { A tomada de decisão baseada na observação do } \\
\text { passado é mais eficaz do que uma tomada de } \\
\text { decisão baseada em métodos preditivos. }\end{array}$ & \multirow{4}{*}{$\begin{array}{l}\text { (Arasteh et al., } \\
\text { 2011); } \\
\text { (Cokins, 2013); } \\
\text { (Delen, 2014); } \\
\text { (McAfee et al., } \\
\text { 2012) }\end{array}$} \\
\hline & $\begin{array}{l}\text { Quando a tomada de decisão é baseada em } \\
\text { métodos preditivos a decisão final está menos } \\
\text { sujeita a falhas. }\end{array}$ & \\
\hline & $\begin{array}{l}\text { A informação é um dos ativos mais importantes } \\
\text { de uma empresa, desse modo é importante que } \\
\text { tenhamos acesso à informação mais correta } \\
\text { possível. }\end{array}$ & \\
\hline & $\begin{array}{l}\text { O Enterprise Resource Planning (ERP), e a } \\
\text { sua componente de gestão operacional, } \\
\text { colaborativa e analítica dos clientes conhecida } \\
\text { por Customer Relationship Management } \\
\text { (CRM), são recursos relativamente comuns nas } \\
\text { empresas. Qual destes recursos utiliza mais } \\
\text { como suporte à tomada de decisão? }\end{array}$ & \\
\hline \multirow{5}{*}{$\begin{array}{l}\text { Expectativa do } \\
\text { Esforço/Preço }\end{array}$} & Preço & \multirow{5}{*}{$\begin{array}{l}\text { (Arasteh et al., } \\
\text { 2011); } \\
\text { (Cokins, 2013); } \\
\text { (Delen, 2014); } \\
\text { (McAfee et al., } \\
\text { 2012) }\end{array}$} \\
\hline & Mudanças necessárias na organização & \\
\hline & Retorno do Investimento & \\
\hline & Segurança e Privacidade & \\
\hline & Tempo necessário para a implementação & \\
\hline \multirow{3}{*}{ Influência Social } & $\begin{array}{l}\text { Irá a Inteligência Artificial eliminar mais } \\
\text { trabalhos do que aqueles que irá criar? }\end{array}$ & \multirow{3}{*}{$\begin{array}{l}\text { (Ford, 2015); } \\
\text { (Jarrahi, 2018) }\end{array}$} \\
\hline & $\begin{array}{l}\text { A Inteligência Artificial ajuda apenas na } \\
\text { automação dos processos. }\end{array}$ & \\
\hline & $\begin{array}{l}\text { A Inteligência Artificial deve ser usada apenas } \\
\text { na automação dos processos. }\end{array}$ & \\
\hline
\end{tabular}

\subsection{Definição das Hipóteses}

As hipóteses foram desenvolvidas através da análise de literatura e o Quadro III apresenta-as em síntese. 


\section{Quadro III}

Definição das Hipóteses

\begin{tabular}{l}
\hline \multicolumn{1}{c}{ Hipóteses } \\
\hline H1: A falta de mão de obra especializada \\
é inibidora da implementação de Business \\
Analytics.
\end{tabular}

(Appelbaum et al., 2017);

(Delen, 2014);

(Erffmeyer \& Johnson, 2001);

(McAfee et al., 2012);

(Mendoza et al., 2007);

(Payne \& Frow, 2006)

(Mesquita et al., 2013);

H2: Em mercados complexos existe (Rascao, 2006); maior perceção das vantagens.

(Riccio, 1992);

(Shuai et al., 2007)

(Erffmeyer \& Johnson, 2001);

H3: Diferente nível de gestão tem diferente perceção das vantagens.

(Mendoza et al., 2007);

(Mesquita et al., 2013);

(Payne \& Frow, 2006);

(Riccio, 1992)

H4: A cultura organizacional é um dos principais obstáculos à implementação, ou seja, quando a gestão é mais baseada intuição a implementação de Business

(Arasteh et al., 2011);

(Cokins, 2013);

(Delen, 2014) Analytics é mais difícil.

H5: O preço é um dos principais
inibidores da adoção do Business
Analytics.

(Delen, 2014);

(McAfee et al., 2012)

H6: As mudanças na organização é um dos principais inibidores da adoção do Business Analytics.

(Delen, 2014);

(McAfee et al., 2012)

H7: A incerteza no retorno do investimento é um dos principais (Delen, 2014); inibidores da adoção do Business (McAfee et al., 2012) Analytics.

H8: A segurança e a privacidade são um dos principais inibidores da adoção do Business Analytics.

(Delen, 2014);

(McAfee et al., 2012)

H9: O tempo necessário à implementação é um dos principais inibidores da adoção do Business Analytics.

(Delen, 2014);

(McAfee et al., 2012) 


\section{ANÁLISE DE RESULTADOS}

\subsection{População e Amostra}

O processo de recolha de dados foi obtido através da realização de um inquérito por questionário online, cujo público alvo foi a população portuguesa. O total de respostas foi de 102, sendo que 47,8\% dos inquiridos se identificam como indivíduos do sexo Masculino e 52,2\% como sendo do sexo Feminino.

\subsection{Discussão de Resultados}

Antes de qualquer análise é importante recordar uma vez mais, que o objetivo do presente estudo foi a identificação das motivações que suportam a adoção de ferramentas de BA pelas organizações portuguesas. De modo a atingir o objetivo proposto, utilizou-se uma análise exploratória que teve como base no modelo da UTAUT.

A UTAUT prevê sete determinantes de aceitação: Expectativa de Desempenho, Expectativa de Esforço, Influência Social, Condições Facilitadoras, Intenção Comportamental, Hábito e Preço.

A identificação dos determinantes de aceitação foi possível através da utilização de uma análise por componentes principais que procura encontrar representações simplificadas de um conjunto de dados correlacionados entre si e, deste modo, interpretar as principais fontes de variabilidade. A análise rotaciona o conjunto de pontos em torno da média, e posteriormente organiza as componentes através da comparação das variâncias.

No entanto, a análise realizada apenas permitiu a identificação de quatro determinantes de aceitação: Expectativa de Desempenho, Expectativa de Esforço, Condições Facilitadoras e a Intenção Comportamental. O Quadro IV apresenta o resultado da análise, onde na primeira coluna se identificaram as perceções dos sujeitos relativamente a diferentes variáveis para as quatro dimensões identificadas. 


\section{Quadro IV}

Análise em Componentes Principais

\begin{tabular}{|c|c|c|c|c|}
\hline Variáveis & $\begin{array}{c}\text { Dimensão } \\
1\end{array}$ & $\begin{array}{c}\text { Dimensão } \\
2\end{array}$ & $\begin{array}{c}\text { Dimensão } \\
3\end{array}$ & $\begin{array}{c}\text { Dimensão } \\
4\end{array}$ \\
\hline Cultura da Empresa & 2.372 & 14.285 & 0.341 & 0.043 \\
\hline $\begin{array}{l}\text { Competência da Empresa para } \\
\text { Adoção }\end{array}$ & 2.933 & 19.544 & 0.199 & 0.501 \\
\hline $\begin{array}{l}\text { Competência para Analisar } \\
\text { Dados }\end{array}$ & 2.270 & 22.113 & 0.685 & 0.240 \\
\hline $\begin{array}{l}\text { Eliminação de Postos de } \\
\text { Trabalho derivado da IA } \\
\end{array}$ & 0.001 & 1.937 & 19.971 & 0.001 \\
\hline Diferentes utilizações da IA & 0.099 & 0.060 & 12.345 & 16.963 \\
\hline Diferentes vantagens da IA & 0.005 & 0.098 & 18.254 & 16.963 \\
\hline $\begin{array}{l}\text { Vantagens dos métodos } \\
\text { preditivos }\end{array}$ & 0.726 & 0.027 & 9.450 & 0.051 \\
\hline $\begin{array}{l}\text { Diminuição das falhas através } \\
\text { dos métodos preditivos }\end{array}$ & 0.593 & 0.055 & 6.717 & 3.176 \\
\hline Valor da Informação & 2.521 & 0.001 & 0.680 & $\mathbf{1 0 . 1 7 5}$ \\
\hline $\begin{array}{l}\text { Valor do Preço na Tomada de } \\
\text { Decisão }\end{array}$ & 1.062 & 1.293 & 4.012 & 3.764 \\
\hline $\begin{array}{l}\text { Abertura para a Mudança na } \\
\text { Empresa }\end{array}$ & 3.491 & 1.664 & 4.249 & 5.119 \\
\hline $\begin{array}{l}\text { Importância do Retorno do } \\
\text { Investimento }\end{array}$ & 3.847 & 3.284 & 6.171 & 5.905 \\
\hline $\begin{array}{l}\text { Valor dado à Segurança e da } \\
\text { Privacidade }\end{array}$ & 1.539 & 1.151 & 5.361 & 10.062 \\
\hline Valor dado ao Tempo & 0.302 & 0.023 & 0.330 & 0.028 \\
\hline $\begin{array}{l}\text { Capacidade do BA em gerar um } \\
\text { maior envolvimento }\end{array}$ & 9.320 & 0.023 & 0.330 & 0.028 \\
\hline $\begin{array}{l}\text { Capacidade do BA em gerar um } \\
\text { maior envolvimento }\end{array}$ & 9.116 & 3.482 & 3.491 & 1.994 \\
\hline $\begin{array}{l}\text { Capacidade do BA influenciar a } \\
\text { competitividade }\end{array}$ & 8.885 & 0.003 & 1.293 & 0.022 \\
\hline $\begin{array}{l}\text { Capacidade do BA na melhoria } \\
\text { das métricas }\end{array}$ & 10.622 & 1.822 & 1.775 & 2.449 \\
\hline $\begin{array}{l}\text { Capacidade do BA na melhoria } \\
\text { do desempenho }\end{array}$ & 11.931 & 0.476 & 0.001 & 0.393 \\
\hline $\begin{array}{l}\text { Capacidade do BA no } \\
\text { melhoramento na captação de } \\
\text { novos clientes }\end{array}$ & 8.962 & .095 & 0.407 & .011 \\
\hline $\begin{array}{l}\text { Capacidade do BA melhorar a } \\
\text { comunicação }\end{array}$ & 5.845 & 4.925 & 0.731 & 1.375 \\
\hline
\end{tabular}

O primeiro determinante encontrado está relacionado com a valorização do Desempenho, isto é, corresponde a um indivíduo que valoriza principalmente o desempenho das ferramentas, neste sentido, a 
adoção das ferramentas de Business Analytics e Business Intelligence está dependente das vantagens geradas, ao nível de melhorias de performance assim como ao nível das melhorias no lucro. Espera-se que o indivíduo que se encontre neste perfil apresente um nível mais executivo na organização.

O Quadro V apresenta, em resumo, o resultado da regressão clássica efetuada para as quatro dimensões, onde foi possível verificar que a valorização do desempenho é tanto maior quanto maior for a idade do indivíduo, o que vai de encontro ao esperado, pois normalmente as posições executivas são ocupadas por indivíduos mais velhos nas empresas tradicionais.

Em Portugal, a formação académica dos gestores de muitas empresas ainda se encontra ao nível do ensino secundário, por esse motivo é normal que uma das variáveis identificadas neste determinante esteja relacionada com esse fator.

Analogamente o segundo determinante, corresponde às condições facilitadoras à adoção, isto é, a capacidade que a empresa onde o indivíduo se encontra tem de análise e gestão de dados, mostra que empresas mais avançadas, neste âmbito, tornam os indivíduos mais abertos para a adoção de ferramentas de Business Analytics.

Verificou-se que, quanto maior o envolvimento dentro da organização maior será a probabilidade de a organização adotar ferramentas de Business Analytics, como tinha sido antecipado por Delen (2014).

Autores como Arasteh et al. (2011), Cokins (2013) e Delen (2014) tinham identificado que um dos principais obstáculos à implementação era a cultura organizacional, deste modo, era de esperar que indivíduos em empresas que valorizam o envolvimento e uma cultura organizacional aberta, estejam mais predispostos a adotar o BA.

A inexistência de mão-de-obra especializada é também comprometedora, como já tinha sido adiantado por Appelbaum et al. (2017), Delen (2014) Erffmeyer \& Johnson (2001), McAfee et al. (2012), Mendoza et al. (2007) e Payne \& Frow (2006).

O terceiro determinante encontrado está relacionado com a Expectativa do Esforço, para estes indivíduos a adoção do Business Analytics depende do tempo necessário para a implementação, da 
segurança, da informação que irão conseguir obter e ainda do conhecimento que têm ou não da inteligência artificial.

Deste modo, podemos assumir que os indivíduos que dão mais valor a este determinante se encontram numa posição na organização mais tática. Verificou-se uma maior propensão de adoção por parte de indivíduos do sexo masculino.

No entanto, a nossa amostra está um pouco enviesada pois foram obtidas maior número de respostas de homens em posição de decisão tática do que mulheres.

A literatura, mais concretamente, Delen (2014) e McAfee et al. (2012), antecipava que as mudanças na organização, o tempo e a segurança seriam os dos principais impedimentos à adoção. Todavia, os mesmo autores avançavam ainda com a questão do preço e do retorno como impeditivos, contudo esses motivos não foram considerados pelos inquiridos do presente estudo.

O último determinante encontrado, está relacionado com a perceção do indivíduo relativamente à Inteligência Artificial, e por sua vez, relacionado com a determinante da Influência Social.

Verificou-se que a propensão para adotar ferramentas de Business Analytics diminui com a idade, podemos então assumir que são as gerações mais novas as mais preparadas e conscientes das vantagens da Inteligência Artificial.

Adicionalmente foi ainda possível observar que são os homens, e indivíduos de departamentos de IT e Desenvolvimento de Produto, os mais abertos à adoção, o que vai de acordo com o esperado pois são estas pessoas as mais informadas sobre as vantagens da Inteligência Artificial.

Retomado as hipóteses antecipadas, é agora possível concluir, que as motivações para adoção de Business Analytics dos indivíduos portugueses estão dependentes de diversos fatores, como anteriormente antecipado.

Investigadores como Erffmeyer \& Johnson (2001), Mendoza et al. (2007), Mesquita et al. (2013), Payne \& Frow (2006) e Riccio (1992) anteciparam que a perceção das vantagens seria diferente para os diversos níveis de gestão; deste modo não é surpreendente que os diferentes determinantes, desempenho, esforço e a intenção comportamental, estejam relacionadas a diferentes níveis hierárquicos. 
O mercado em Portugal é relativamente pequeno, não existe grande heterogeneidade, por consequência não foi possível percecionar uma diferença de vantagens relacionada com o tipo de mercado, como tinha sido adiantado por Mesquita et al. (2013), Rascão (2006), Riccio (1992) e Shuai et al. (2007).

Em conclusão, podemos assumir que as motivações relativas à adoção do Business Analytics dependem principalmente da posição hierárquica em que a pessoa esteja inserida. Ainda se verificou que o preço e o retorno do investimento, não são considerados impedimentos na adoção. No entanto, o não conhecimento, em especial o não conhecimento da inteligência artificial parecem ser os principais problemas dos sujeitos inquiridos. 


\section{Quadro V}

\section{Regressão Clássica}

\begin{tabular}{|c|c|c|c|c|}
\hline Variáveis & Dimensão 1 & Dimensão 2 & Dimensão 3 & Dimensão 4 \\
\hline Idade 18-25 & Significante. & $\mathrm{n} / \mathrm{a}$ & $\mathrm{n} / \mathrm{a}$ & Significante. \\
\hline Idade 26-40 & Significante. & $\mathrm{n} / \mathrm{a}$ & $\mathrm{n} / \mathrm{a}$ & Significante. \\
\hline Idade 41-65 & $\mathrm{n} / \mathrm{a}$ & $\mathrm{n} / \mathrm{a}$ & $\mathrm{n} / \mathrm{a}$ & Significante. \\
\hline Sexo Masculino & $\mathrm{n} / \mathrm{a}$ & $\mathrm{n} / \mathrm{a}$ & Significante. & Significante. \\
\hline Sexo Feminino & $\mathrm{n} / \mathrm{a}$ & $\mathrm{n} / \mathrm{a}$ & $\mathrm{n} / \mathrm{a}$ & $\mathrm{n} / \mathrm{a}$ \\
\hline $\begin{array}{l}\text { Administração e } \\
\text { Financeiro }\end{array}$ & $\mathrm{n} / \mathrm{a}$ & $\mathrm{n} / \mathrm{a}$ & $\mathrm{n} / \mathrm{a}$ & $\mathrm{n} / \mathrm{a}$ \\
\hline Executivo & $\mathrm{n} / \mathrm{a}$ & $\mathrm{n} / \mathrm{a}$ & $\mathrm{n} / \mathrm{a}$ & $\mathrm{n} / \mathrm{a}$ \\
\hline Gestão do Produto & $\mathrm{n} / \mathrm{a}$ & $\mathrm{n} / \mathrm{a}$ & $\mathrm{n} / \mathrm{a}$ & Significante. \\
\hline IT & $\mathrm{n} / \mathrm{a}$ & $\mathrm{n} / \mathrm{a}$ & $\mathrm{n} / \mathrm{a}$ & $\mathrm{n} / \mathrm{a}$ \\
\hline Marketing & $\mathrm{n} / \mathrm{a}$ & $\mathrm{n} / \mathrm{a}$ & $\mathrm{n} / \mathrm{a}$ & $\mathrm{n} / \mathrm{a}$ \\
\hline Operações / Logística & $\mathrm{n} / \mathrm{a}$ & $\mathrm{n} / \mathrm{a}$ & $\mathrm{n} / \mathrm{a}$ & $\mathrm{n} / \mathrm{a}$ \\
\hline $\begin{array}{l}\text { Desenvolvimento do } \\
\text { Produto }\end{array}$ & $\mathrm{n} / \mathrm{a}$ & $\mathrm{n} / \mathrm{a}$ & $\mathrm{n} / \mathrm{a}$ & Significante. \\
\hline Recursos Humanos & $\mathrm{n} / \mathrm{a}$ & $\mathrm{n} / \mathrm{a}$ & $\mathrm{n} / \mathrm{a}$ & $\mathrm{n} / \mathrm{a}$ \\
\hline Serviço ao Cliente & $\mathrm{n} / \mathrm{a}$ & $\mathrm{n} / \mathrm{a}$ & $\mathrm{n} / \mathrm{a}$ & $\mathrm{n} / \mathrm{a}$ \\
\hline Vendas & $\mathrm{n} / \mathrm{a}$ & $\mathrm{n} / \mathrm{a}$ & $\mathrm{n} / \mathrm{a}$ & $\mathrm{n} / \mathrm{a}$ \\
\hline $\begin{array}{l}\text { Banca/Finanças/Segur } \\
\text { os }\end{array}$ & $\mathrm{n} / \mathrm{a}$ & $\mathrm{n} / \mathrm{a}$ & $\mathrm{n} / \mathrm{a}$ & $\mathrm{n} / \mathrm{a}$ \\
\hline $\begin{array}{l}\text { Ciências da } \\
\text { Vida/Saúde }\end{array}$ & $\mathrm{n} / \mathrm{a}$ & $\mathrm{n} / \mathrm{a}$ & $\mathrm{n} / \mathrm{a}$ & $\mathrm{n} / \mathrm{a}$ \\
\hline $\begin{array}{l}\text { Retalho/Venda por } \\
\text { Grosso }\end{array}$ & $\mathrm{n} / \mathrm{a}$ & $\mathrm{n} / \mathrm{a}$ & $\mathrm{n} / \mathrm{a}$ & $\mathrm{n} / \mathrm{a}$ \\
\hline Infraestruturas & $\mathrm{n} / \mathrm{a}$ & $\mathrm{n} / \mathrm{a}$ & $\mathrm{n} / \mathrm{a}$ & $\mathrm{n} / \mathrm{a}$ \\
\hline Produtos Industriais & $\mathrm{n} / \mathrm{a}$ & $\mathrm{n} / \mathrm{a}$ & $\mathrm{n} / \mathrm{a}$ & $\mathrm{n} / \mathrm{a}$ \\
\hline Serviços & $\mathrm{n} / \mathrm{a}$ & $\mathrm{n} / \mathrm{a}$ & $\mathrm{n} / \mathrm{a}$ & $\mathrm{n} / \mathrm{a}$ \\
\hline $\begin{array}{l}\text { Tecnologia/ Media/ } \\
\text { Telecomunicações }\end{array}$ & $\mathrm{n} / \mathrm{a}$ & $\mathrm{n} / \mathrm{a}$ & $\mathrm{n} / \mathrm{a}$ & $\mathrm{n} / \mathrm{a}$ \\
\hline Educação Básica & $\mathrm{n} / \mathrm{a}$ & $\mathrm{n} / \mathrm{a}$ & $\mathrm{n} / \mathrm{a}$ & $\mathrm{n} / \mathrm{a}$ \\
\hline Educação Secundária & Significante. & $\mathrm{n} / \mathrm{a}$ & $\mathrm{n} / \mathrm{a}$ & $\mathrm{n} / \mathrm{a}$ \\
\hline Licenciatura & $\mathrm{n} / \mathrm{a}$ & $\mathrm{n} / \mathrm{a}$ & $\mathrm{n} / \mathrm{a}$ & $\mathrm{n} / \mathrm{a}$ \\
\hline Mestrado & $\mathrm{n} / \mathrm{a}$ & $\mathrm{n} / \mathrm{a}$ & $\mathrm{n} / \mathrm{a}$ & $\mathrm{n} / \mathrm{a}$ \\
\hline Doutoramento & $\mathrm{n} / \mathrm{a}$ & $\mathrm{n} / \mathrm{a}$ & $\mathrm{n} / \mathrm{a}$ & $\mathrm{n} / \mathrm{a}$ \\
\hline $\begin{array}{l}\text { Aumento do } \\
\text { Envolvimento }\end{array}$ & $\mathrm{n} / \mathrm{a}$ & Significante & $\mathrm{n} / \mathrm{a}$ & $\mathrm{n} / \mathrm{a}$ \\
\hline $\begin{array}{l}\text { Melhorias de } \\
\text { Produtividade }\end{array}$ & $\mathrm{n} / \mathrm{a}$ & $\mathrm{n} / \mathrm{a}$ & $\mathrm{n} / \mathrm{a}$ & $\mathrm{n} / \mathrm{a}$ \\
\hline $\begin{array}{l}\text { Otimização de } \\
\text { Operações }\end{array}$ & $\mathrm{n} / \mathrm{a}$ & $\mathrm{n} / \mathrm{a}$ & $\mathrm{n} / \mathrm{a}$ & $\mathrm{n} / \mathrm{a}$ \\
\hline $\begin{array}{l}\text { Transformação dos } \\
\text { Produtos }\end{array}$ & $\mathrm{n} / \mathrm{a}$ & $\mathrm{n} / \mathrm{a}$ & $\mathrm{n} / \mathrm{a}$ & $\mathrm{n} / \mathrm{a}$ \\
\hline
\end{tabular}




\section{CONCLUSÕES}

O objetivo central focou-se na identificação das motivações para a adoção de ferramentas de BA na população portuguesa. Neste sentido, foi possível identificar quatro determinantes de aceitação: a expectativa de desempenho, do esforço as condições facilitadoras e a influência social.

Através da definição das determinantes, conseguimos verificar uma ligeira ligação entre estes e os diferentes níveis hierárquicos de uma organização, isto é, verificou-se que alguém num nível hierárquico superior valoriza principalmente o desempenho da ferramenta, em detrimento do esforço e das condições.

Foi possível apurar que, uma empresa que promova o envolvimento aumenta a probabilidade de sucesso na adoção das ferramentas de Business Analytics e Business Intelligence.

Concluiu-se também, que a propensão para adotar ferramentas de Business Analytics e Business Intelligence diminui com a idade, ou seja, verificamos que são as gerações mais novas são as mais preparadas e conscientes das vantagens. Neste sentido, empresas com colaboradores mais novos são mais abertas para as novas ferramentas.

Adicionalmente, foi ainda possível observar que são os homens, e indivíduos de departamentos de IT e Desenvolvimento de Produto os mais abertos à adoção, o que vai de acordo com o esperado, pois assume-se que são estas pessoas as mais informadas sobre as suas vantagens.

Todavia, verificou-se que o preço e o respetivo retorno financeiro não são os fatores mais importantes, ao contrário do que tinha sido avançado pela literatura.

No entanto, a conclusão mais importante foi a identificação do não conhecimento e do medo da inteligência artificial como os principais condicionantes à adoção.

Não obstante, tratando-se de um estudo exploratório não foi possível, ainda assim administrá-lo num contexto mais adequado, como junto de empresas a quadros específicos das mesmas, de diferentes setores e dimensão.

Num trabalho futuro seria recomendado, além da aplicação do estudo junto de um maior número de pessoas com ligação à economia e à gestão, 
adotar a entrevista e a análise de conteúdo para aprofundar e explicar os resultados obtidos.

Deste modo, uma das principais limitações do estudo foi 0 enviesamento da amostra, derivado do facto de a maioria dos inquiridos se encontrar na área das tecnologias e/ou em posições que envolvem o conhecimento das ferramentas e dos principais algoritmos.

\section{REFERÊNCIAS BIBLIOGRÁFICAS}

Acito, F., \& Khatri, V. (2014). Business analytics: Why now and what next? Business Horizons, 57(5), 565-570.

https://doi.org/10.1016/j.bushor.2014.06.001

Amaral, L. (1994). PRAXIS: um referencial para o planeamento de sistemas de informação. Retrieved from http://repositorium.sdum.uminho.pt/handle/1822/49

Appelbaum, D., Kogan, A., Vasarhelyi, M., \& Yan, Z. (2017). Impact of business analytics and enterprise systems on managerial accounting. International Journal of Accounting Information Systems, 25, 29-44. https://doi.org/10.1016/j.accinf.2017.03.003

Arasteh, A., Aliahmadi, A., Mahmoodi, H. S., \& Mohammadpour, M. O. (2011). Role of information technology in business revolution. The International Journal of Advanced Manufacturing Technology, 53(1), 411-420. https://doi.org/10.1007/s00170-010-2834-9

Chen, Chiang, \& Storey. (2012). Business Intelligence and Analytics: From Big Data to Big Impact. MIS Quarterly, 36(4), 1165. https://doi.org/10.2307/41703503

Cokins, G. (2013). Top 7 trends in management accounting. Strategic Finance, 95(6), 21-30.

Coming of Age Digitally: Learning, Leadership and Legacy | Deloitte Insights. (2018). Retrieved February 27, 2019, from https://www2.deloitte.com/insights/us/en/focus/digitalmaturity/coming-of-age-digitally-learning-leadership-legacy.html

Cooper, A. (2012). What is Analytics? Definition and Essential Characteristics.

Cordeiro, S., \& Gouveia, L. B. (2018). Regulamento Geral de Proteção de Dados (RGPD): o novo pesadelo das empresas? [Report]. Retrieved from *TRS Tecnologia, Redes e Computadores website: https://bdigital.ufp.pt/handle/10284/6714 
Delen, D. (2014). Real-World Data Mining: Applied Business Analytics and Decision Making - Dursun Delen - Google Livros. Retrieved February 27, 2019, from https://books.google.pt/books?hl=ptPT\&lr=\&id=O_nbBQAAQBAJ\&oi=fnd\&pg=PR6\&dq=22.\%09Dele n,+D.+(2014).+Realworld+data+mining:+applied+business+analytics+and+decision+ma king.+FT+Press.\&ots=McFdnnu-RZ\&sig $=\mathrm{CT} 8$ rkq6rDTxAXSv2fT32xjCFbI\&redir_esc $=y \# v=$ onepage $\& q=22 . \% 09 \mathrm{D}$ elen\%2C\%20D.\%20(2014).\%20Real-

world $\% 20$ data $\% 20$ mining $\% 3 \mathrm{~A} \% 20$ applied $\% 20$ business $\% 20$ analytics $\% 20$ and \%20decision \%20making.\%20FT\%20Press.\&f=false

Erffmeyer, R. C., \& Johnson, D. A. (2001). An exploratory study of sales force automation practices: expectations and realities. Journal of Personal Selling \& Sales Management, 21(2), 167-175.

Ferreira, S. A., \& Andrade, A. (2013). Desenhar e implementar um sistema de learning analytics no ensino superior. Gestão e Desenvolvimento, 21, 123-146.

Ford, M. (2015). Rise of the Robots: Technology and the Threat of a Jobless Future. Basic Books.

Ghosh, A., Gajar, P. K., \& Rai, S. (2013). Bring Your Own Device (BYOD): Security Risks and Mitigating Strategies. Journal of Global Research in Computer Science, 4(4), 62-70.

Jarrahi, M. H. (2018). Artificial Intelligence and the Future of Work. Kaye, D. (1995). Sources of information, formal and informal. Management Decision, 33(5), 13-15.

Lindgreen, A., Hingley, M. K., Grant, D. B., \& Morgan, R. E. (2012). Value in business and industrial marketing: Past, present, and future. Industrial Marketing Management, 41(1), 207-214.

McAfee, A., Brynjolfsson, E., Davenport, T. H., Patil, D. J., \& Barton, D. (2012). Big data: the management revolution. Harvard Business Review, 90(10), 60-68.

Mendoza, L. E., Marius, A., Pérez, M., \& Grimán, A. C. (2007). Critical success factors for a customer relationship management strategy. Information and Software Technology, 49(8), 913-945.

Mesquita, V., Faria, J., Gonçalves, D., \& Varajão, J. (2013). Motivations for the adption of ERP and CRM systems: a comparative analysis. 10th International Conference on Information Systems and Technology Management-CONTECSI, 1291-1301. Tecsi. 
Passos, C. (2017). A ética-como motor da inovação empresarial e da sustentabilidade organizacional. Gestão e Desenvolvimento, 25, 5573.

Payne, A., \& Frow, P. (2006). Customer relationship management: from strategy to implementation. Journal of Marketing Management, 22(12), 135-168.

Ransbotham, S., \& Kiron, D. (2017). Analytics as a Source of Business Innovation. 19.

Rascao, J. P. (2006). Da Gestão Estratégica à Gestão Estratégica da Informação: Como aumentar o tempo disponível para a tomada de decisão estratégica. Editora E-papers.

Riccio, E. L. (1992). Uma contribuição ao estudo da contabilidade como sistema de informação (Text, Universidade de São Paulo). https://doi.org/10.11606/T.12.1992.tde-19012009-121736

Santos, M. Y., \& Ramos, I. (2006). Como tornar o seu negócio realmente competitivo: desafios tecnológicos e de gestão.

Shuai, J. J., Yi- Fen Sue, \& Chyan Yang. (2007). The impact of ERP implementation on corporate supply chain performance. 2007 IEEE International Conference on Industrial Engineering and Engineering Management, 1644-1648. https://doi.org/10.1109/IEEM.2007.4419471

Venkatesh, V., Thong, J. Y. L., \& Xu, X. (2012). Consumer Acceptance and Use of Information Technology: Extending the Unified Theory of Acceptance and Use of Technology (SSRN Scholarly Paper No. ID 2002388). Retrieved from Social Science Research Network website: https://papers.ssrn.com/abstract=2002388 